\title{
DIE EUROPÄISCH-AFRIKANISCHE ASSOZIIERUNG UND DIE LATEINAMERIKANISCHEN DRIT'TLÄNDER
}

Jede neue Maßnahme der Assoziierungspolitik der Europäischen Wirtschaftsgemeinschaft (EWG) findet stets auch außerhalb des Kreises der Mitglieds- und assoziierten Länder, also in den sogenannten Drittländern, ein Echo. Das gilt in besonderem Maße von den im Juli 1968 in Kinshasa aufgenommenen Gesprächen über die Fortsetzung der Assoziierungspolitik nach Ablauf des Assoziierungsabkommens von Jaunde am 31. Mai 19691.

Die folgenden Ausführungen über das Verhältnis der Drittländer zur europäischafrikanischen Assoziierung bleiben auf die lateinamerikanischen Länder beschränkt, also auf die größte Gruppe von Drittländern, die gewisse einheitliche Merkmale der geographischen Lage, der Wirtschaftsstruktur und der Sprache aufweisen und für die gegenwärtig keine praktischen Möglichkeiten erkennbar sind, sich selbst in irgendeiner Form an die EWG anzuschließen.

Von Anfang an hat die auf die Assoziierung einer wachsenden Gruppe afrikanischer Länder gerichtete Politik der EWG in Lateinamerika ein kritisches Echo gefunden. Die Kritik der lateinamerikanischen Drittländer an der Assoziierungspolitik fußt vornehmlich auf Artikel 110 des EWG-Vertrages, der die Absicht der Mitgliedstaaten zum Ausdruck bringt, "durch die Schaffung einer Zollunion ... im gemeinsamen Interesse zur harmonischen Entwicklung des Welthandels ... beizutragen“. In die Zollunion sind nach Artikel 132 des EWG-Vertrages sowie Artikel 2 des Jaunde-Abkommens auch die assoziierten Länder eingeschlossen. Die Begünstigung der assoziierten Länder in Afrika insbesondere durch Zollpräferenzen, so wird in Lateinamerika argumentiert, könne nicht ohne Wirkung auf die internationalen Handelsströme bleiben und stehe mithin in klarem Widerspruch zu der in Artikel 110 zum Ausdruck gebrachten Absicht der Gemeinschaft.

Diesem Argument kann indessen entgegengehalten werden, daß die EWG durchaus eine überdurchschnittliche Steigerung des Warenverkehrs innerhalb des Kreises der Mitglieds- und assoziierten Länder anstrebt und als vereinbar mit dem Wortlaut des Artikels 110 ansieht. So wird zum Beispiel in Artikel 2 des EWG-Vertrages als Aufgabe des Zusammenschlusses herausgestellt, „engere Beziehungen zwischen den Staaten zu fördern, die in dieser Gemeinschaft zusammengeschlossen sind", und in Artikel 1 des Jaunde-Vertrages wird „die Erweiterung des Handels zwischen den assoziierten Staaten und den Mitgliedstaaten" der EWG als wichtiges Ziel der Assoziierung bezeichnet. Noch deutlicher kommt die beabsichtigte Bevorzugung der Assoziierten in Artikel 131 des EWG-Vertrages zum Ausdruck, nach dem „die Assoziierung in erster Linie den Interessen der Einwohner dieser (assoziierten) Länder und Hoheitsgebiete dienen“ soll. Daraus ergibt sich, daß als „harmonische Entwicklung des Welthandels" keinesfalls die starre Beibehaltung der vor Gründung der EWG gegebenen regionalen Aufteilung des Gesamtimports der sechs EWG-Länder $\mathrm{zu}$ verstehen ist, sondern lediglich die Vermeidung einer ausgesprochen disharmonischen Weiterentwicklung des internationalen Güteraustausches.

Störungen dieser Art hat die gemeinsame Agrarpolitik der EWG, insbesondere die EWG-Marktordnung für Rindfleisch, verursacht; zutreffend äußerte Bundesaußenminister Brandt am 12. Dezember 1968 in einer Rede vor den Botschaftern der lateinamerikanischen Länder in Bonn, daß „als Folge der europäischen Agrarpolitik in der Struktur unserer Einfuhren aus Lateinamerika Verschiebungen eingetreten" seien. Dagegen sind aber derartige Störungen nach den Erfahrungen der vergangenen Jahre von der Assoziierungspolitik kaum zu erwarten. Es erscheint zwar unmöglich, den Einfluß der Zollpräferenzen für tropische Agrarprodukte aus

1 Siehe hierzu auch Verfassung und Recht in Obersee 1968, S. $453 \mathrm{ff}$. 
assoziierten afrikanischen Ländern auf den Import der sechs EWG-Länder exakt zu ermitteln; sicherlich haben die Zollvorteile im afrikanisch-lateinamerikanischen Wettbewerb zugunsten der assoziierten Länder in Afrika gewirkt, wie es ihr Zweck ist; ebenso sicher ist es aber auch, daß eine ausgesprochen disharmonische Entwicklung der EWG-Einfuhr von Kaffee, Bananen, Kakao und anderen tropischen Erzeugnissen aus Lateinamerika als Folge der Assoziierungspolitik bisher nicht zu beobachten war. Der Kaffeeimport der EWG aus Lateinamerika erhöhte sich von 303,6 Millionen US-Dollar (342 119 t) 1960 auf 400,7 Millionen US-Dollar (460 057 t) im Jahre 1967; der Anteil Lateinamerikas an der gesamten wertmäßigen Kaffeeeinfuhr der EWG hat sich in der genannten Zeitspanne nahezu unverändert gehalten (1960: 60,4 Prozent; 1967: 59,5 Prozent). Sogar noch günstigere Tendenzen zeigt die Bananeneinfuhr seit 1960: Im Jahre 1960 betrug der Bananenimport der EWG aus Lateinamerika 64,7 Millionen US-Dollar (541615 t), 1967 dagegen bereits 146,4 Millionen US-Dollar (995 310 t); der Beitrag Lateinamerikas zum gesamten Bananenimport der EWG stieg von 43 Prozent 1960 auf 55 Prozent 1967.

Das am 16. Juli 1966 in Lagos mit Nigeria sowie auch das am 27. Juli 1968 in Arusha mit den ostafrikanischen Ländern Kenia, Uganda und Tansania unterzeichnete Assoziierungsabkommen enthalten überdies Vorschriften, die in der Zukunft der Vermeidung von größeren Strukturänderungen im internationalen Handel dienen sollen. So schreibt das Abkommen von Arusha zum Beispiel vor, daß die Zollfreiheit für Kaffeeeinfuhr aus den genannten ostafrikanischen Ländern auf die Menge beschränkt bleiben soll, die der jährlichen Durchschnittseinfuhr der Gemeinschaft aus diesen Ländern in den letzten drei Jahren entspricht. Diese Bestimmung bezweckt zwar den Schutz wirtschaftlicher Interessen anderer assoziierter Länder, sie begünstigt aber gleichzeitig auch die Kaffeeländer in Lateinamerika.

Ein weiterer Anlaß zu heftiger Kritik seitens der lateinamerikanischen Drittländer ist die Erweiterung des Kreises der assoziierten Länder. Offenbar hatte sich in lateinamerikanischen Fachkreisen die Vorstellung festgesetzt, daß Artikel 131 des EWG-Vertrages gewissermaßen als "numerus clausus" für die für eine Assoziierung in Betracht kommenden Länder zu gelten habe. Nach Artikel 131 sollen „die außereuropäischen Länder und Hoheitsgebiete, die mit Belgien, Frankreich, Italien und den Niederlanden besondere Beziehungen unterhalten“, der Gemeinschaft assoziiert werden. Die sogenannten Alt-Assoziierten waren in der Tat ausschließlich Länder, die zu einem Mitgliedsland der EWG „besondere Beziehungen“ unterhielten, d. h. in einem Abhängigkeitsverhältnis irgendeiner Art zu Belgien, Frankreich oder Italien entweder gestanden hatten oder im Zeitpunkt des Inkrafttretens der ersten Assoziierungsvereinbarung noch standen. Anläßlich der Unterzeichnung des Assoziierungsabkommens in Jaunde am 20. Juli 1963 wurde sodann aber seitens der EWG die Absicht erklärt, mit weiteren Drittländern über die Herstellung engerer Beziehungen zu verhandeln. Als erstes Commonwealth-Land schloß, wie schon erwähnt, im Juli 1966 Nigeria ein Assoziierungsabkommen besonderer Art mit der Gemeinschaft, und im Juli 1968 folgten die ebenfalls zum Commonwealth of Nations gehörenden ostafrikanischen Länder Kenia, Uganda und Tansania dem Beispiel Nigerias.

Diese sogenannte Neu-Assoziierung entspricht vollauf den rechtlichen Grundlagen der Integration. Artikel 238 des EWG-Vertrages sowie auch Artikel 58 des JaundeAbkommens sehen die Assoziierung weiterer Länder über den in Artikel 131 des EWG-Vertrages abgegrenzten Kreis hinaus ausdrücklich vor. Ferner konnte die EWG-Kommission lateinamerikanische Kritiker darauf hinweisen, daß die Assoziierungspolitik in der Vergangenheit keinesfalls spektakuläre Änderungen der internationalen Handelsströme verursacht habe und daß eine solche Wirkung künftig infolge der Verringerung der EWG-Außenzölle, die ja pari passu eine Verminderung der Präferenzmarge bewirkt, immer weniger zu befürchten sei. Dennoch hat sich aber mit der Ausdehnung des Kreises der assoziierten Länder über die soge- 
nannten "Assoziierten der ersten Stunde“ hinaus die Resonanz, die dieses İnstrument der EWG-Außenpolitik in Lateinamerika fand, grundlegend geändert. Während sich objektive lateinamerikanische Kreise von Anfang an bemüht zeigten, für die nach Artikel 131 des EWG-Vertrages geschlossenen Assoziierungsabkommen Verständnis aufzubringen, zumal diese Vereinbarungen als Ausdruck besonderen Verantwortungsbewußtseins bestimmter EWG-Mitgliedsländer gegenüber ihren ehemaligen Einflußgebieten in Afrika angesehen werden können, lösten die späteren Assoziierungsverhandlungen mit Nigeria sowie mit Kenia, Uganda und Tansania erbitterte Kritik in Lateinamerika, insonderheit in den Kaffee und Kakao produzierenden lateinamerikanischen Ländern, aus.

Obgleich also, wie gezeigt wurde, die Assoziierungspolitik der EWG weder mit aus den zugrundeliegenden Verträgen hergeleiteten rechtlichen noch mit aus der Entwicklung des Welthandels hergeleiteten ökonomischen Argumenten angreifbar erscheint, haben sich in Lateinamerika mit jeder neuen Assoziierungsvereinbarung Skepsis und Kritik vergrößert. Die Grundlage dieser Kritik ist, auch wenn sie sich vornehmlich ökonomischer Argumente zu bedienen bemüht, hauptsächlich psychologischer Art. Sie erklärt sich zum Teil aus der wirtschaftlichen Angewiesenheit lateinamerikanischer Rohstoffländer auf die westeuropäischen Absatzmärkte, zum Teil aus dem Bestreben Lateinamerikas, eine allzu starke wirtschaftliche Orientierung auf die USA durch Ausbau der Kontakte mit Westeuropa zu verhindern, und schließlich wohl auch aus den in Europa immer wieder geäußerten Beteuerungen der historisch gewachsenen europäisch-lateinamerikanischen Verbundenheit, die sich aus lateinamerikanischer Sicht mit einer Ausrichtung der EWG auf die afrikanischen Entwicklungsländer schlecht in Einklang bringen lassen. Als ökonomisches Argument gegen die europäisch-afrikanische Assoziierung, auf das in späterem Zusammenhang noch eingegangen werden soll, bleibt schließlich die Unvereinbarkeit der Gewährung von Zollpräferenzen an eine begrenzte Gruppe von Ländern mit dem Streben nach einem weltweiten Präferenzsystem zugunsten der Gesamtheit der Entwicklungsländer.

Nachstehend sollen einige lateinamerikanische Stimmen zur Integration Westeuropas und insbesondere zur europäisch-afrikanischen Assoziierung wiedergegeben werden ${ }^{2}$ :

Eine besonders klare Formel für die Sorgen und Erwartungen, die Lateinamerika an die wirtschaftliche Integration Westeuropas knüpft, hat schon im Jahre 1957 der damalige uruguayische Botschafter in Bonn, Carlos A. Clulow, gefunden: „Unsere Länder erwarten ... mit gutem Recht als Mitglieder der großen Völkerfamilie, daß ihre Belange nicht übergangen werden, daß sie nicht vergessen werden in den Plänen für die Neuordnung der Welt von morgen." Am 18. Juli 1961 ließ die Regierung Argentiniens anläßlich einer Konferenz der Regierungschefs der EWGMitgliedsländer in Bonn ein Memorandum überreichen, in dem es hieß, die europäische Integration habe ausgesprochen bevorzugte Wirtschaftsräume entstehen lassen, von denen in wachsendem Ausmaß ungünstige Auswirkungen auf die Agrarausfuhrländer Lateinamerikas ausgingen. Im Juli 1962 berief die Comisión Económica para América Latina (CEPAL) eine Expertengruppe nach Santiago de Chile, die den Auftrag erhielt, eine Studie ${ }^{3}$ über die Möglichkeiten einer Koordinierung der Politik der lateinamerikanischen Länder gegenüber der EWG zu erarbeiten. Diesem Expertenteam gehörten der heutige argentinische Wirtschaftsminister, Adalbert Krieger Vasena, der damalige uruguayische Botschafter in Bonn, Julio A. Lacarte Muró, sowie der Chilene Pedro Daza und der Brasilianer José Garrido Torres an. Bezeichnend für die auch heute noch in Lateinamerika verbreitete öffentliche

2 H. J. Hartmann und J. Westphalen: Europa auf falschem Kurs? Lateinamerikanische EWG-Sorgen, Hamburg 1963.

3 Hacia la Coordinación de la Política Comercial de América Latina. Las Relaciones con la Comunidad Económica Europea. Análisis y recomendaciones del grupo de consultores convocado por la Secretaría, herausgegeben von CEPAL, Santiago de Chile 1962. 
Meinung über die EWG ist der folgende Auszug aus dieser Studie: „Die ausgeprägte Prosperität, die sich in den letzten Jahren in Westeuropa und insbesondere in den EWG-Mitgliedsländern entwickelt hat, bedeutet - insgesamt betrachtet recht wenig für die Ausfuhr Lateinamerikas. Daran zeigt sich, daß das bekannte Argument, die Prosperität Europas begünstige direkt auch Lateinamerika, ... keine ausreichende Bestätigung in der Praxis findet."

Diese Behauptung ist heute mit den statistischen Gesamtergebnissen der Außenhandelsentwicklung klar zu widerlegen. Die Gesamteinfuhr der Gemeinschaft aus Lateinamerika hat sich von 1,65 Mrd. US-Dollar im Jahre 1958 auf 2,74 Mrd. US-Dollar im Jahre 1967 erhöht. Allerdings darf man sich durch diese günstigen Gesamtergebnisse nicht darüber hinwegtäuschen lassen, daß sich die EWG-Einfuhr bestimmter Agrarprodukte aus der gemäßigten Klimazone Lateinamerikas ungünstig entwickelt hat. Daraus läßt sich begründete Kritik an bestimmten Instrumenten der Integration herleiten; keinesfalls wäre es aber zulässig, aus solchen isolierten Wirkungen, so schwerwiegend sie im Einzelfall auch sein mögen, grundsätzliche Zweifel an der Ausstrahlung des wirtschaftlichen Aufschwungs in Westeuropa auf die Außenwelt zu folgern.

Die Tatsache, daß die zitierte Außerung aus der CEPAL-Studie auch heute noch in lateinamerikanischen Fachkreisen zu hören ist, läßt erkennen, wie fest die Vorurteile gegenüber der europäischen Integration in der öffentlichen Meinung Lateinamerikas verwurzelt sind. Daraus ist aber zu folgern, daß die Kritik der Lateinamerikaner an der europäisch-afrikanischen Assoziierung, auch wenn sie der juristischen und ökonomischen Grundlage entbehren mag, eine ernste Belastung der europäisch-lateinamerikanischen Beziehungen darstellt.

In jüngster Zeit haben die Unterzeichnung des Arusha-Abkommens und die Gespräche von Kinshasa den lateinamerikanischen Sorgen wieder neue Nahrung gegeben. Ende Juni 1968 fand in Santo Domingo auf Ministerebene eine Konferenz der Comisión Especial de Coordinación Latinoamericana (CECLA) statt, die sich sowohl mit dem damals im Endstadium der Vorverhandlungen befindlichen Assoziierungsabkommen mit den drei ostafrikanischen Staaten als auch mit dem Thema der einen knappen Monat später beginnenden Tagung von Kinshasa, also den Grundsatzfragen der künftigen Assoziierungspolitik, befaßte und in der Resolución 10/68 M die Stellungnahme Lateinamerikas zu diesen Fragen formulierte. In der CECLA-Resolution wird besonders hervorgehoben, daß die Kritik Lateinamerikas an der europäisch-afrikanischen Assoziierung keinesfalls gleichgestellt werden dürfte mit einer Opposition gegen die Bemühungen der EWG um die wirtschaftliche Entwicklung der assoziierten Länder in Afrika. Jedoch dürfe sich die Assoziierungspolitik keiner Maßnahmen der Handelsdiskriminierung zum Nachteil Lateinamerikas bedienen. Ferner wird auf die Schwierigkeiten hingewiesen, die sich aus der Beibehaltung regional begrenzter Zollpräferenzen für die Schaffung eines allgemeinen, weltweiten Präferenzsystems zu Gunsten der Fertig- und Halbfertigprodukte aus Entwicklungsländern gemäß den Beschlüssen der letzten Welthandelskonferenz ergeben müssen. Dieser Gedanke verdient, besonders hervorgehoben zu werden: Angesichts der Bemühungen um eine weltweite Lösung der Probleme des internationalen Handels mutet der Abschluß von Freihandelsvereinbarungen zwischen der EWG und einzelnen afrikanischen Entwicklungsländern in der Tat anachronistisch an.

In Europa ist die lateinamerikanische Kritik an der EWG und speziell an der Assoziierungspolitik nicht ohne Echo geblieben. Nachstehend werden einige Beispiele für europäische bzw. deutsche Außerungen zu diesem Fragenkreis angeführt:

Schon im April 1958 hatten die Regierungen der in der EWG zusammengeschlossenen Länder in dem Bestreben, einer mit Sicherheit erwarteten Kritik zuvorzukommen, ein Memorandum an die Regierungen der lateinamerikanischen Länder gerichtet, das Professor Hallstein ein "wirtschaftspolitisches Friedensangebot" 
genannt hat. In diesem Memorandum sind in großen Zügen die Ziele der Gemeinschaft dargelegt und ein Meinungsaustausch mit allen Ländern Lateinamerikas angeboten worden. Anfang 1962 legte die EWG-Kommission dem Ministerrat ein "Aktionsprogramm für Lateinamerika" vor, in dem u.a. die Einberufung von Zusammenkünften der bei der EWG akkreditierten lateinamerikanischen Botschafter mit Vertretern der Kommission und die Errichtung von Informationsbüros der Gemeinschaft in wichtigen lateinamerikanischen Hauptstädten vorgeschlagen wurde. Die erstgenannte Maßnahme, also die Schaffung des sogenannten Kontaktausschusses, ist vom Ministerrat in seiner Sitzung am 30./31. Mai 1963 beschlossen worden, und auch die Errichtung von Informationsbüros ist inzwischen sowohl in Montevideo als auch in Santiago de Chile erfolgt. Durch diese Maßnahmen hat indessen nicht vermieden werden können, daß in Lateinamerika mit jedem neuen Schritt der Assoziierungspolitik Mißtrauen und Vorurteile gegenüber der EWG wieder aufleben.

Die bisherigen Bemühungen der EWG um den lateinamerikanischen Subkontinent hat der derzeitige Präsident der Kommission der Europäischen Gemeinschaften, Jean Rey, in einer am 11. Juli 1968 in Hamburg gehaltenen Rede ${ }^{4}$ mit bemerkenswerter Selbstkritik zusammengefaßt: „Seit Jahren sind wir im Gespräch mit den lateinamerikanischen Ländern. ... Wir haben uns regelmäßig mit den Botschaftern zu Gesprächen getroffen. Wir haben vor allem versucht, in der Kennedy-Runde etwas zu tun. Es war nicht ohne Erfolg, aber es war bescheiden, ganz bescheiden. ... Die Zeit ist m. E. gekommen, wieder in direkten Kontakten mit den lateinamerikanischen Staaten zu einem besseren Zusammenwirken zwischen ihnen und der Gemeinschaft zu kommen."

Für die Zukunft bieten sich vor allem die folgenden Ansatzpunkte für Maßnahmen der EWG zur Besserung ihrer Beziehungen zu Lateinamerika.

Erstens erscheint es erforderlich, seitens der EWG-Kommission den direkten Kontakt zu den Regierungen der lateinamerikanischen Länder aufzunehmen und auf höchster Ebene das Gespräch über die Lateinamerika interessierenden Instrumente der europäischen Integrationspolitik zu suchen. Als Vertretung der am Außenhandel mit Lateinamerika beteiligten deutschen Wirtschaftskreise hat der Ibero-Amerika Verein am 21. Juni 1968, also vor den Konferenzen von Kinshasa und Arusha, bei der EWG-Kommission angeregt, alle bestehenden Absichten auf dem Gebiet der Assoziierungspolitik den lateinamerikanischen Regierungen offenzulegen, um damit der Entstehung neuer Vorurteile in Lateinamerika und neuer psychologischer Belastungen der europäisch-lateinamerikanischen Beziehungen vorzubeugen. In einer solchen Erklärung müßte die Kommission „überzeugend zum Ausdruck bringen, daß sie nach Kräften bemüht ist, ihre Verpflichtungen gegenüber den assoziierten Ländern im Einklang zu halten mit den berechtigten Anliegen der lateinamerikanischen Drittländer".

Zweitens erscheint es angebracht, die möglichen wirtschaftlichen Auswirkungen der Assoziierungspolitik auf Lateinamerika dadurch in immer engere Grenzen zu zwängen, daß die Außenzölle der Gemeinschaft konsequent weiter abgebaut werden. Diese Forderung hat die von den Spitzenverbänden der deutschen Wirtschaft getragene "Arbeitsgemeinschaft Entwicklungsländer" in ihrer Stellungnahme vom 12. August $1968^{5}$ mit folgenden Worten klar zum Ausdruck gebracht: „In der deutschen Wirtschaft ist man ... der Ansicht, daß die schon mit Abschluß des Jaunde-Abkommens eingeleitete Tendenz, die Präferenzmarge für tropische Produkte durch Herabsetzungen bzw. Aussetzungen des gemeinsamen Zolltarifs zu verringern, in dem neuen Abkommen konsequent weiter verfolgt werden sollte mit dem Ziel, den regionalen Präferenzraum allmählich ganz einzuebnen.“

\section{JÜRGEN WESTPHALEN}

\footnotetext{
4 J. Rey, Die Rolle und die Verantwortlichkeiten der Gemeinschaft in der Welt, in: Der Ubersee-Club Hamburg, Mitteilungen (Juli 1968), S. $5 \mathrm{ff}$.

5 Siehe hierzu auch Verfassung und Recht in Ubersee 1968, S. 459.
} 